LAWRENCE LIVERMORE N A TIO N A L LABORATORY
General computational spectroscopic framework applied to Z-pinch dynamic hohlraum K-shell argon spectra

M. L. Adams, D. B. Sinars, H. A. Scott

January 14, 2005

Journal of Quantitative Spectroscopy \& Radiative Transfer 
This document was prepared as an account of work sponsored by an agency of the United States Government. Neither the United States Government nor the University of California nor any of their employees, makes any warranty, express or implied, or assumes any legal liability or responsibility for the accuracy, completeness, or usefulness of any information, apparatus, product, or process disclosed, or represents that its use would not infringe privately owned rights. Reference herein to any specific commercial product, process, or service by trade name, trademark, manufacturer, or otherwise, does not necessarily constitute or imply its endorsement, recommendation, or favoring by the United States Government or the University of California. The views and opinions of authors expressed herein do not necessarily state or reflect those of the United States Government or the University of California, and shall not be used for advertising or product endorsement purposes. 


\title{
General computational spectroscopic framework applied to Z-pinch dynamic hohlraum K-shell argon spectra
}

\author{
M.L. Adams ${ }^{a, *}$, D.B. Sinars ${ }^{b}$, and H.A. Scott ${ }^{a}$ \\ ${ }^{a}$ Lawrence Livermore National Laboratory, Livermore, CA 94550, USA \\ ${ }^{\mathrm{b}}$ Sandia National Laboratories, Albuquerque, NM 87185, USA
}

\begin{abstract}
We describe a general computational spectroscopic framework for interpreting observed spectra. The framework compares synthetic spectra with measured spectra, then optimizes the agreement using the DAKOTA toolkit to minimize a merit function that incorporates established spectroscopic techniques. We generate synthetic spectra using the self-consistent nonlocal thermodynamic equilibrium atomic kinetics and radiative transfer code CRETIN, relativistic atomic structure and cross section data from Hullac, and detailed spectral line shapes from Totalb. We test the capabilities of both our synthetic spectra model and general spectroscopic framework by analyzing a K-shell argon spectrum from a Z-pinch dynamic hohlraum inertial confinement fusion capsule implosion experiment. The framework obtains close agreement between an experimental spectrum measured by a time integrated focusing spectrometer and the optimal synthetic spectrum. The synthetic spectra show that considering the spatial extent of the capsule and including the effects of optically thick resonance lines significantly affects the interpretation of measured spectra.
\end{abstract}

Key words: plasma spectroscopy, plasma diagnostic techniques, X-ray spectra, Z-pinch dynamic hohlraum experiments

PACS: 32.30.Rj, 52.58.Lq, 52.70.-m

\footnotetext{
* University of California, Lawrence Livermore National Laboratory, L-018, 7000 East Ave., Livermore, CA 94550, USA

Email address: mladams@llnl.gov (M.L. Adams).
} 


\section{Introduction}

Plasma spectroscopy is the science of determining plasma properties through the observation and interpretation of emergent radiation. In this paper we develop a general computational framework to facilitate the interpretation of spectra. We then use this framework to analyze K-shell argon spectra from a Z-pinch dynamic hohlraum experiment done on the Z-Machine at Sandia National Laboratories [1].

Two approaches are commonly taken to interpret spectra. In the first approach, a spectral signature from a dominant physical process is used to identify system properties. Most approaches of this type determine a single property, e.g., chemical composition from the quantum fingerprints of atoms and molecules in the system or electron density from the full-width at halfmaximum (FWHM) of a spectral line shape. While this approach requires several assumptions regarding the plasma, e.g., homogeneity or optically thin, it often produces results that are within experimental diagnostic uncertainties. However, the underlying assumptions are frequently not justified and the interpretation of the results may be suspect. A second approach involves the generation of synthetic spectra. The reliability of this approach depends on the applicability, or capabilities, of the model used to generate the synthetic spectra, as well as the method used to determine and obtain agreement between synthetic and measured spectra. In what follows, we develop a general spectroscopic framework that combines both approaches.

Background radiation and debris make plasma spectroscopy on Z-pinch dynamic hohlraum experiments challenging, compared to laser driven inertial confinement fusion (ICF) experiments. Recent advances in radiation sources, resulting from improved wire array performance [2], and developments in diagnostic capabilities [3], have made Z-pinch experiments a popular spectroscopic playground. Thus, to test the capabilities of our synthetic spectra model and general spectroscopic framework, we analyze K-shell argon spectra from a Zpinch dynamic hohlraum ICF capsule implosion experiment.

The remainder of this paper conforms to the following outline. Section 2 establishes the general spectroscopic framework. Section 3 covers the generation of synthetic spectra pertaining to Z-pinch dynamic hohlraum ICF capsule implosions. Section 4 applies the spectroscopic framework to Z-pinch dynamic hohlraum time-integrated K-shell argon spectra measured using a focusing spectrometer with spatial resolution. Finally, Section 5 summarizes the paper and discusses possible directions for future research. 


\section{General spectroscopic framework}

Interpreting plasma spectra often requires either large amounts of time or the development tailored algorithms for each application. To both automate the interpretation of plasma spectra and allow for simple adaptability, we have constructed a general computational framework for performing plasma spectroscopy based on the DAKOTA toolkit [4].

Designed as an engineering tool for finding extremum solutions, the DAKOTA toolkit includes several optimization algorithms and a noninvasive interface to outside computational models. The interface works by passing design variables to a computational model and returning evaluations of user-specified merit functions, or objective functions, to the DAKOTA toolkit. Using a prescribed optimization algorithm, the DAKOTA toolkit then determines the optimal design variables by minimizing the merit function. To perform plasma spectroscopy, this interface is applied to a computational model for generating synthetic spectra and extended to include subroutines that implement established spectroscopic techniques.

Given a set of plasma properties, i.e., the design variables, the spectroscopic interface constructs the following merit function:

$$
f=\frac{1}{N} \sum_{i=1}^{N} W_{i}\left(y_{m, i}-y_{s, i}\right)^{2}
$$

where $y$ is a discrete value used to compare measured, $m$, and synthetic, $s$, spectra, $N$ is the total number of values, and $W$ is a weighting parameter. While common choices for $y$ include the ratio of spectral line intensities and the FWHM of a spectral line shape [5], any relevant quantity can be used. For example, $y$ could represent the specific intensity at a given frequency or correspond to another objective function.

Once a merit function has been established, the DAKOTA toolkit applies one of several optimization algorithms to minimize the merit function and hence determine the optimal set of plasma properties. For example, the DAKOTA toolkit includes nongradient-based global search techniques like the genetic algorithm. When a large number of parallel processors are available, we have found that a simple parameter study, i.e., the straightforward procedure of evaluating merit functions on an organized grid of points chosen to span the plasma property domain, obtains the optimum solution in the least amount of time. For example, let us imagine there are 3 plasma properties, 200 parallel processors, and that 10 points suffice to map out each plasma property domain. Then there are a total of $10^{3}$ points in this plasma property space and only 5 iterations per processor are necessary for optimization. Very few optimization algorithms 
can converge in less than 5 iterations, therefore the parameter study requires the least amount of real time.

For plasma spectroscopy applications, the parameter study has the following additional advantages over other optimization algorithms. First, when a unique minimum does not exist, the parameter study clearly identifies all possible solutions. Gradient-based methods simply fail when they encounter multiple solutions and nongradient-based methods require excessive iterations to map out the interesting plasma property space. Second, for repeated spectroscopy applications, once the computationally expensive portion of the optimization is performed, namely the generation of synthetic spectra, this information is saved and can be reused. This enables the study of variations in the merit function and rapid analysis of different experimental spectra.

\section{Dynamic hohlraum capsule synthetic spectra model}

Z-pinch dynamic hohlraums can generate radiation sources with sufficient energy to implode an inertial confinement fusion (ICF) capsule $[6,7,3]$. In the dynamic hohlraum concept, cylindrically imploding high-Z (high-opacity) plasmas impact low-density (low-opacity) cylindrical foam converters. This impact launches a radiative shock towards the axis and implodes a spherical ICF capsule. Similar to indirect drive laser and ion beam ICF experiments, the fusion fuel filled Z-pinch capsules are frequently doped with an impurity that is convenient for spectroscopic observation. Characteristics of the capsule implosion and radiation source are then obtained from spectroscopic observation and interpretation of this dopant.

To model the spectra from a Z-pinch dynamic hohlraum ICF capsule, we first assume the capsule is spherically symmetric with radius $R$ (see Fig. 1). Although the cylindrically symmetric radiative shock tends to produce an elongated capsule implosion shape [3], the spherical assumption is a good first approximation when analyzing spectra measured over intervals of space and time. Next we assume the capsule has a constant free electron density and temperature. In the simulations that follow the capsule fuel is $\mathrm{D}_{2}$, the dopant is argon, and we assume quasineutrality. Thus, we can fix the background deuterium ion density, $n_{d}$, by

$$
n_{d}=n_{e}-\bar{Z} n_{i}
$$

where $n_{e}\left(n_{i}\right)$ is the free electron (argon) density and $\bar{Z}$ is the average argon ionic charge, which is chosen to be 17 .

We generate synthetic K-shell argon spectra using the self-consistent nonlo- 
cal thermodynamic equilibrium (NLTE) atomic kinetics and radiative transfer code Cretin [8], relativistic atomic structure and cross section data from Hullac [9], and detailed spectral line shapes from Totalb [10]. For the given capsule configuration, CRETIN calculates the radial variation of argon atomic level populations and the corresponding radiation field. Then the synthetic spectra are generated by calculating the area-integrated spectral intensity escaping the ICF capsule and intersecting a fictional detector (see Fig. 1). Hullac provides atomic structure and cross section data for Ar XIX, Ar XXVIII $(n=1-8)$, Ar XXVII $(n=1-8)$, Ar XXVI $(n=1-8)$, and the dielectronic satellites through $n=5$, where $n$ is the principal quantum number. TotalB spectral line shapes are used in the CREtin line transfer routines for the dominant H-like (Ar XXVIII) and He-like (Ar XXVII) argon lines.

To illustrate this model, we construct synthetic spectrum for a Z-pinch dynamic hohlraum ICF capsule with the following properties: gas fill of 24 atm $\mathrm{D}_{2}$ and 0.085 atm $\mathrm{Ar}$, capsule radius $R=200 \mu \mathrm{m}$, electron density $n_{e}=$ $10^{23} \mathrm{~cm}^{-3}$, and electron temperature $T_{e}=1 \mathrm{keV}$. Figure 2 depicts the spatial variation in argon isoelectronic densities. Figure 3 depicts the K-shell argon spectral intensity as seen by the detector and the corresponding frequencydependent optical depth.

Figure 2 shows that the H-like argon density is larger than the He-like argon density in the capsule core, while the reverse is true at the edge. This variation in isoelectronic densities is accompanied by a decrease in radiation energy density at the edge. The cause of this behavior is the large optical depths of the principal resonance lines of the isoelectronic sequences, i.e., $\operatorname{Ar} \operatorname{Ly}_{\alpha} \sim 30$ and Ar $\mathrm{He}_{\alpha} \sim 40$. At the capsule core the resonance photons drive up the excited level populations, increasing ionization at a given electron temperature. Near the edge, more photons escape the capsule, the radiation energy density in the lines decreases, and ionization decreases at a given electron temperature.

We mention two important points for the interpretation of measured spectra. First, accurately modeling the line transfer of the optically thick principal resonance lines is necessary to obtain the spatial variation of the ionization balance. Second, the radiation for a given spectral frequency originates within an optical depth of the capsule surface. The optically thick frequencies will reflect conditions in the outer layers of the capsule, while the optically thin frequencies represent conditions throughout the capsule. 


\section{K-shell argon spectral data interpretation}

To test the capabilities of both our synthetic spectra model and general spectroscopic framework we analyze a K-shell argon spectrum from a Z-pinch dynamic hohlraum ICF capsule implosion experiment. The argon spectrum was measured using a focusing spectrometer with spatial resolution (FSSR). For more information on the Z-pinch dynamic hohlraum experiment and spectrometer the reader is encouraged to consult Sinars et al. [1].

Given the experimental ICF capsule gas fill (24 atm $\mathrm{D}_{2}$ and $\left.0.085 \mathrm{~atm} \mathrm{Ar}\right)$ and time-integrated FSSR data, we seek to quantify the ICF capsule radius, electron density, and electron temperature. TO apply the spectroscopic framework, we use the synthetic spectra model covered in Sec. 3, discretize the plasma property space for the parameter study discussed in Sec. 2, and define a merit function. The plasma property mesh contains 6048 design points with 8 linear spaced capsule radius points from $50-400 \mu \mathrm{m}, 21$ logarithmically spaced electron density points from $5 \cdot 10^{21}-5 \cdot 10^{23} \mathrm{~cm}^{-3}$, and 36 linearly spaced electron temperature points from $700-1400 \mathrm{eV}$. The upper and lower bounds of the plasma properties were chosen to span the range of possible experimental outcomes, while the resolution of the mesh was chosen to be within diagnostic uncertainties.

We consider several merit functions. Beginning with the simplest, we consider the ratio of $\mathrm{Ar} \mathrm{Ly} \beta$ to $\mathrm{Ar} \mathrm{He}_{\beta}$ line intensities (see Fig. 4a) and the FWHM of the $\mathrm{Ar} \mathrm{He}_{\beta}$ line (see Fig. 4b). Both these techniques have a long history in X-ray plasma spectroscopy and can be used to iteratively determine both the electron temperature and electron density [11]. For example, the nearly vertical behavior of the merit function in Fig. $4 \mathrm{~b}$ indicates that the $\mathrm{Ar} \mathrm{He}_{\beta}$ FWHM has a strong dependence on the electron density. Given this electron density, we could determine the electron temperature from the numerous possible solutions in Fig. 4a. Rather that pursue this approach, we will form a more elaborate merit function.

To interpret the Z-pinch dynamic hohlraum FSSR spectrum, an excellent merit function is formed by taking all possible ratios between the 5 strongest argon spectral line intensities, i.e., $\mathrm{Ly}_{\alpha}, \mathrm{Ly}_{\beta}, \mathrm{Ly}_{\gamma}, \mathrm{He}_{\beta}$, and $\mathrm{He}_{\gamma}$. This merit function yields the bulls eye pattern centered on the optimal ICF capsule plasma properties, i.e., capsule radius $R=200 \mu \mathrm{m}$, electron density $n_{e}=7.9 \cdot 10^{22} \mathrm{~cm}^{-3}$, and electron temperature $T_{e}=900 \mathrm{eV}$ (see Fig. 5). Figure. 6 compares the synthetic and measured spectra. Since the measured spectra is time-integrated and spatially averaged, we consider this to be close agreement between the spectra. The relative intensities of the spectral lines are in quantitative agreement and several other spectral features are in qualitative agreement, e.g., the

simulated structure of the $\mathrm{Ar} \mathrm{He}_{\beta}$ Li-like satellites is similar to that in the 
measured spectrum.

\section{Summary and discussion}

This paper described a general computational spectroscopic framework for interpreting measured spectra. The framework generates synthetic spectra using the NLTE atomic kinetics and radiative transfer code CRETIN, relativistic atomic structure and cross section data from Hullac, and detailed spectral line shapes from TOTALB. The synthetic spectra are compared with measured spectra using merit functions that encapsulate established spectroscopic techniques. Finally, the framework optimizes the agreement with the measured spectra by using the DAKOTA toolkit. The framework can easily incorporate numerous spectroscopic methods and can be rapidly applied to multiple sets of measured spectra.

It is common practice in plasma spectroscopy to assume uniform and homogeneous plasmas to interpret spectra, i.e., a zero dimensional (0D) approximation. To move beyond $0 \mathrm{D}$, our synthetic spectra incorporate a finite spatial extent and allow atomic level populations and the radiation field to vary selfconsistently within a spherical ICF capsule. For systems with optically thick dominant lines, this produces a more realistic spectrum, which translates to an improved interpretation of measured spectra.

As an application of the spectroscopic framework, we analyzed K-shell argon spectra from a Z-pinch dynamic hohlraum ICF capsule implosion experiment using synthetic spectra from a simple spherical capsule configuration. The optimal synthetic line intensities agreed quantitatively with those of the measured spectrum and many other spectral features, e.g., intensities of satellite and continuum radiation, agreed qualitatively. Most of the discrepancies between the spectra can be attributed to the space and time averaged nature of the measured data, but the disagreement near the argon He-like resonance continuum edge could indicate a limitation of our synthetic spectra model.

Future applications of this framework to Z-pinch dynamic hohlraums will seek to analyze more sets of experimental data and incorporate more plasma properties, e.g., ion temperature or magnetic field. A major difficulty in achieving the latter extension of the framework is due to the limitation of the parameter study. As the number of design variables increase the parameter study eventually becomes prohibitive and other optimization methods must be considered.

Another issue is the question of interpreting time-integrated and spatiallyaveraged experimental data. Different frequencies will dominate the spectral intensity as time progresses in an experiment, and plasma profiles will evolve 
in time. Both of these effects complicate the interpretation of spectra. Can time-integrated experimental spectra be dissected to yield information about peak implosion conditions? Can observed spectroscopic data be unfolded to yield profile information? While we do not have answers to these questions, we do have a general spectroscopic framework to help address these questions.

\section{Acknowledgments}

This work has relied on numerous python scripts developed by S.T. Brandon and P. Tipton, used atomic data supplied by H.K. Chung, and benefited from spectroscopic discussions with R.W. Lee.

This work was performed under the auspices of the U.S. Department of Energy by the University of California, Lawrence Livermore National Laboratory through contract W-7405-ENG-48.

\section{References}

[1] D.B. Sinars, G.A. Chandler, J.E. Bailey, R.C. Mancini, G.A. Rochau, D.F. Wenger, R.G. Adams, M.L. Adams, H.A. Scott, A.Ya. Faenov, T.A. Pikuz, and S.A. Pikuz. Measurements of K-shell Ar spectra from z-pinch dynamic hohlraum experiments made using a focusing spectrometer with spatial resolution. J. Quant. Spectro. Radiat. Transfer., 2005. [This issue].

[2] D.D. Ryutov, M.S. Derzon, and M.K. Matzen. The physics of fast Z pinches. Rev. Mod. Phys., 72(1):167, 2000.

[3] J.E. Bailey, G.A. Chandler, S.A. Slutz, I. Golovkin, P.W. Lake, J.J. MacFarlane, R.C. Mancini, T.J. Burris-Mog, G. Cooper, R.J. Leeper, T.A. Mehlhorn, T.C. Moore, T.J. Nash, D.S. Nielsen, C.L. Ruiz, D.G. Schroen, and W.A. Varnum. Hot dense capsule-implosion cores produced by Z-pinch dynamic hohlraum radiation. Phys. Rev. Lett., 92(8):085002, 2004.

[4] M.S. Eldred, A.A. Giunta, B.G.B. Waanders, S.F. Wojtkiewicz Jr., W.E. Hart, and M.P. Alleva. DAкотA, A multilevel parallel object-oriented framework for design optimization, parameter estimation, uncertainty quantification, and sensitivity analysis. Technical report, Sandia National Laboratories, 2001. SAND2001-3796.

[5] H.R. Griem. Principles of Plasma Spectroscopy. Cambridge University Press, New York, 1997.

[6] J.E. Bailey, G.A. Chandler, S.A. Slutz, G.R. Bennett, G. Cooper, J.S. Lash, S. Lazier, R. Lemke, T.J. Nash, D.S. Nielson, T.C. Moore, C.L. Ruiz, D.G. 
Schroen, R. Smelser, J. Torres, and R.A. Vesey. X-ray imaging measurements of capsule implosions driven by a Z-pinch dynamic hohlraum. Phys. Rev. Lett., 89(9):095004, 2002.

[7] S.A. Slutz, J.E. Bailey, G.A. Chandler, G.R. Bennett, G. Cooper, J.S. Lash, S. Lazier, P. Lake, R.W. Lemke, T.A. Mehlhorn, T.J. Nash, D.S. Nielson, J. McGurn, T.C. Moore, C.L. Ruiz, D.G. Schroen, J. Torres, W. Varnum, and R.A. Vesey. Dynamic hohlraum driven inertial fusion capsules. Phys. Plasmas, 10(5):1875, 2003.

[8] H.A. Scott. CRetin - A radiative transfer capability for laboratory plasmas. J. Quant. Spectro. Radiat. Transfer., 71(2-6):689, 2001.

[9] A. Bar-Shalom, M. Klapisch, and J. Oreg. Hullac, an integrated computer package for atomic processes in plasmas. J. Quant. Spectro. Radiat. Transfer., $71(2-6): 169,2001$.

[10] M.L. Adams, R.W. Lee, H.A. Scott, H.K. Chung, and L. Klein. Complex atomic spectral line shapes in the presence of an external magnetic field. Phys. Rev. E, $66(6-2): 395,2002$.

[11] J.D. Kilkenny, R.W. Lee, M.H. Key, and J.G. Lunney. X-ray spectroscopic diagnosis of laser-produced plasmas, with emphasis on line broadening. Phys. Rev. A, 2(6):2746, 1980. 


\section{Figure captions}

FIG. 1: Z-pinch dynamic hohlraum ICF capsule geometry. Performing an area integral of the spectral intensity collected by a fictional detector, CRETIN generates synthetic spectra for comparison with measured spectra.

FIG. 2: Spatial variation in H-like (black), He-like (red), and Li-like (green) argon densities. ICF capsule conditions are: gas fill of $24 \mathrm{~atm} \mathrm{D}_{2}$ and $0.085 \mathrm{~atm}$ Ar, capsule radius $R=200 \mu \mathrm{m}$, electron density $n_{e}=10^{23} \mathrm{~cm}^{-3}$, and electron temperature $T_{e}=1 \mathrm{keV}$.

FIG. 3: a) Synthetic K-shell argon spectra and b) corresponding optical depth, $\tau$, of the ICF capsule in Fig. 2.

FIG. 4: Natural logarithm of the merit function obtained from a) the ratio of $\mathrm{Ar} \mathrm{Ly}_{\beta}$ to $\mathrm{Ar} \mathrm{He}_{\beta}$ line intensities (capsule radius is $150 \mu \mathrm{m}$ ) and b) the FWHM of the $\mathrm{Ar} \mathrm{He}_{\beta}$ line (capsule radius is $100 \mu \mathrm{m}$ ).

FIG. 5: Natural logarithm of the merit function formed by taking all possible ratios between the 5 strongest argon spectral line intensities, i.e., $\mathrm{Ly}_{\alpha}, \mathrm{Ly}_{\beta}$, $\mathrm{Ly}_{\gamma}, \mathrm{He}_{\beta}$, and $\mathrm{He}_{\gamma}$. The optimal plasma properties are: $R=200 \mu \mathrm{m}, n_{e}=$ $7.9 \cdot 10^{22} \mathrm{~cm}^{-3}$, and $T_{e}=900 \mathrm{eV}$.

FIG. 6: Comparison between synthetic (black) and measured (red) Z-pinch dynamic hohlraum ICF capsule implosion spectra. ICF capsule conditions are: capsule radius $R=200 \mu \mathrm{m}$, electron density $n_{e}=7.9 \cdot 10^{22} \mathrm{~cm}^{-3}$, and electron temperature $T_{e}=900 \mathrm{eV}$. 


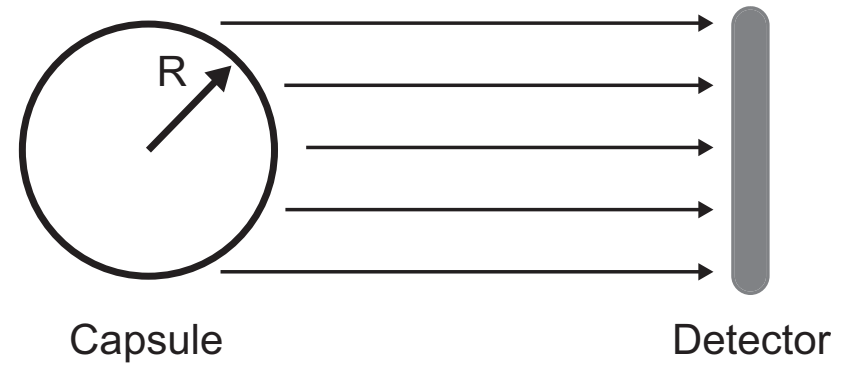

Fig. 1. 


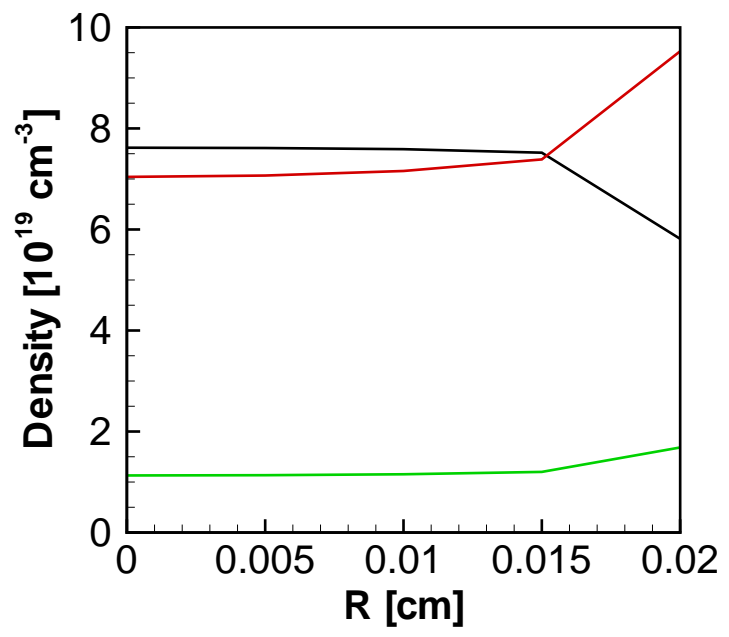

Fig. 2. 


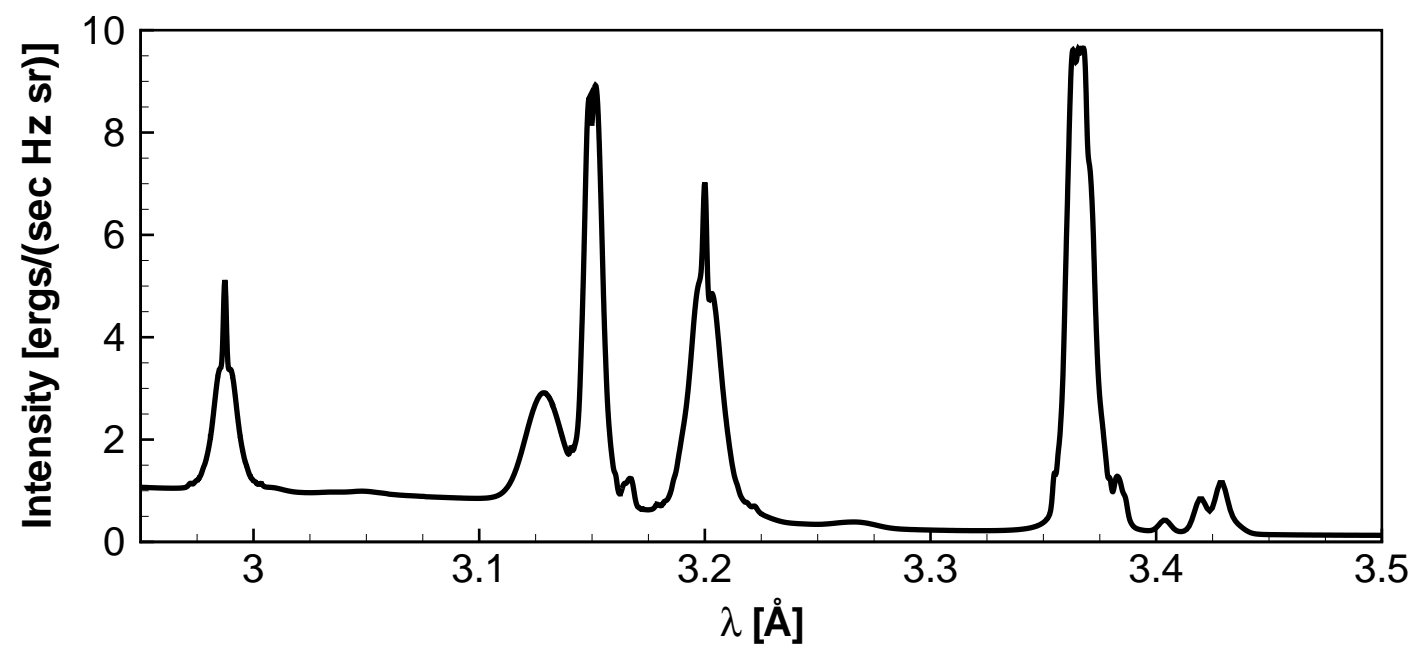

a)

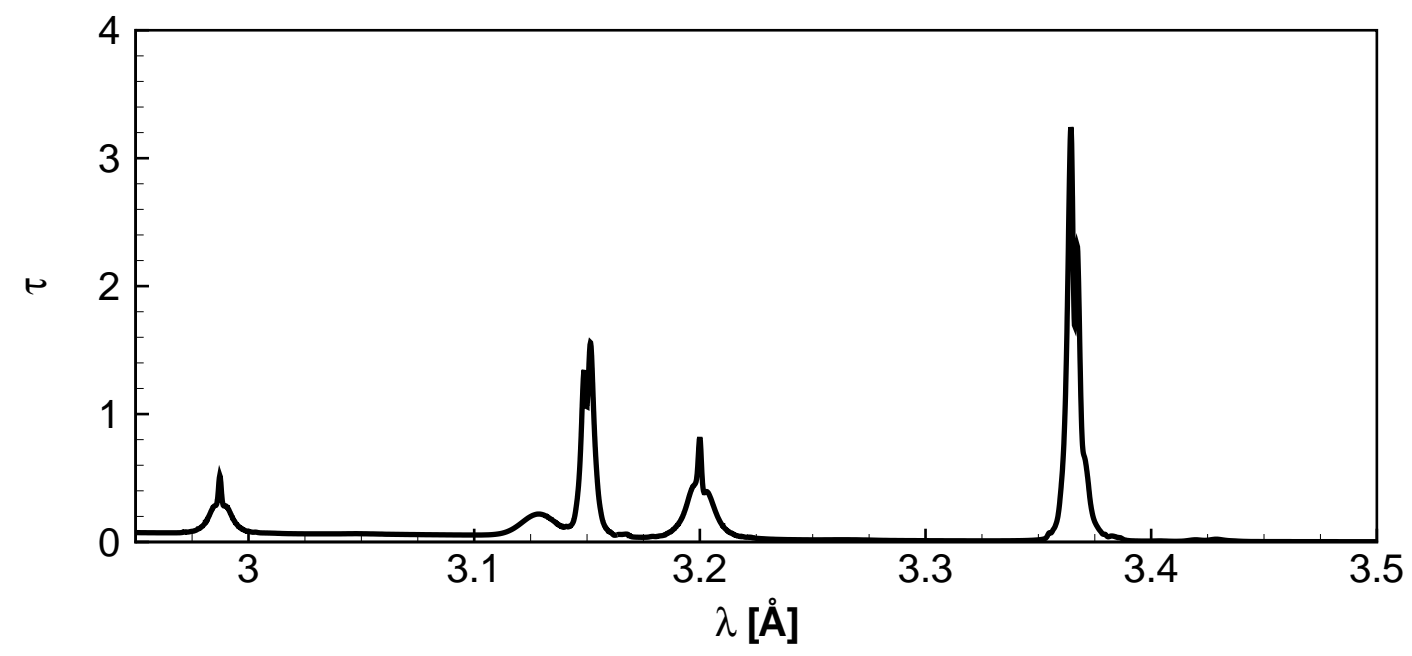

b)

Fig. 3. 

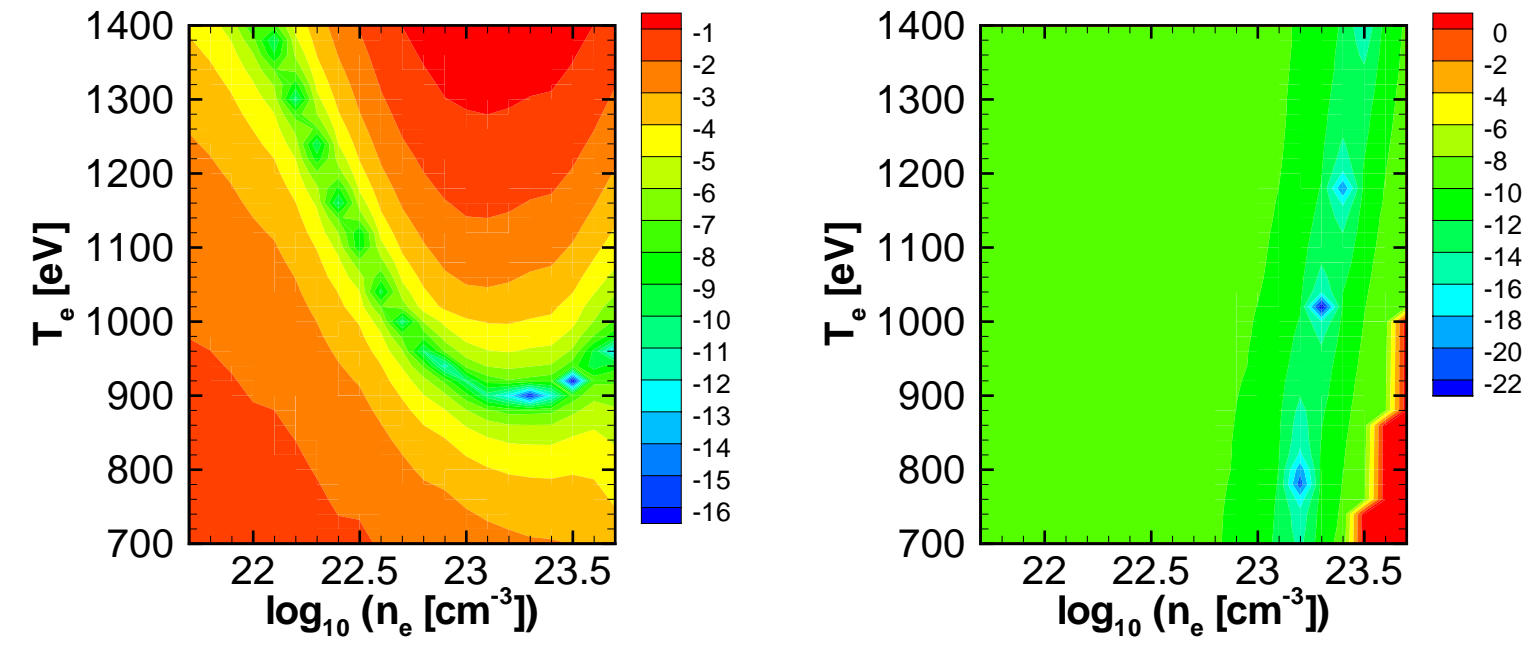

a)

b)

Fig. 4. 


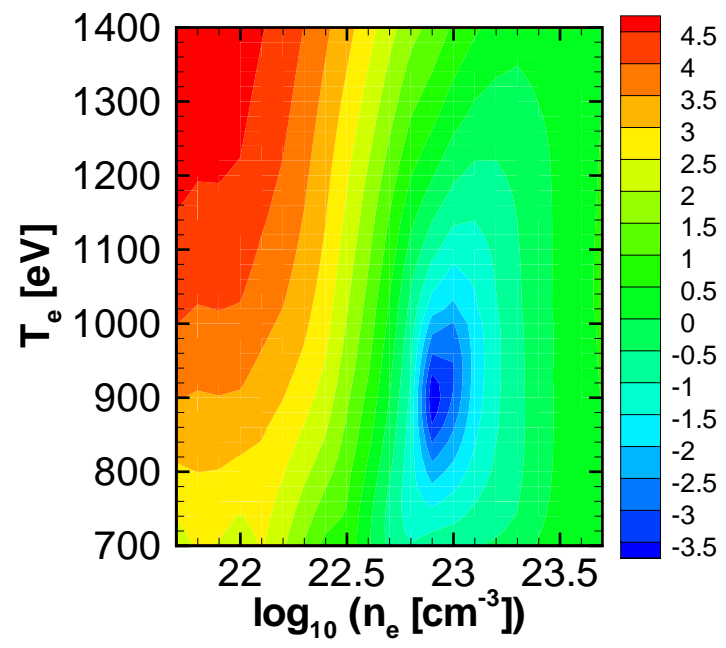

Fig. 5. 


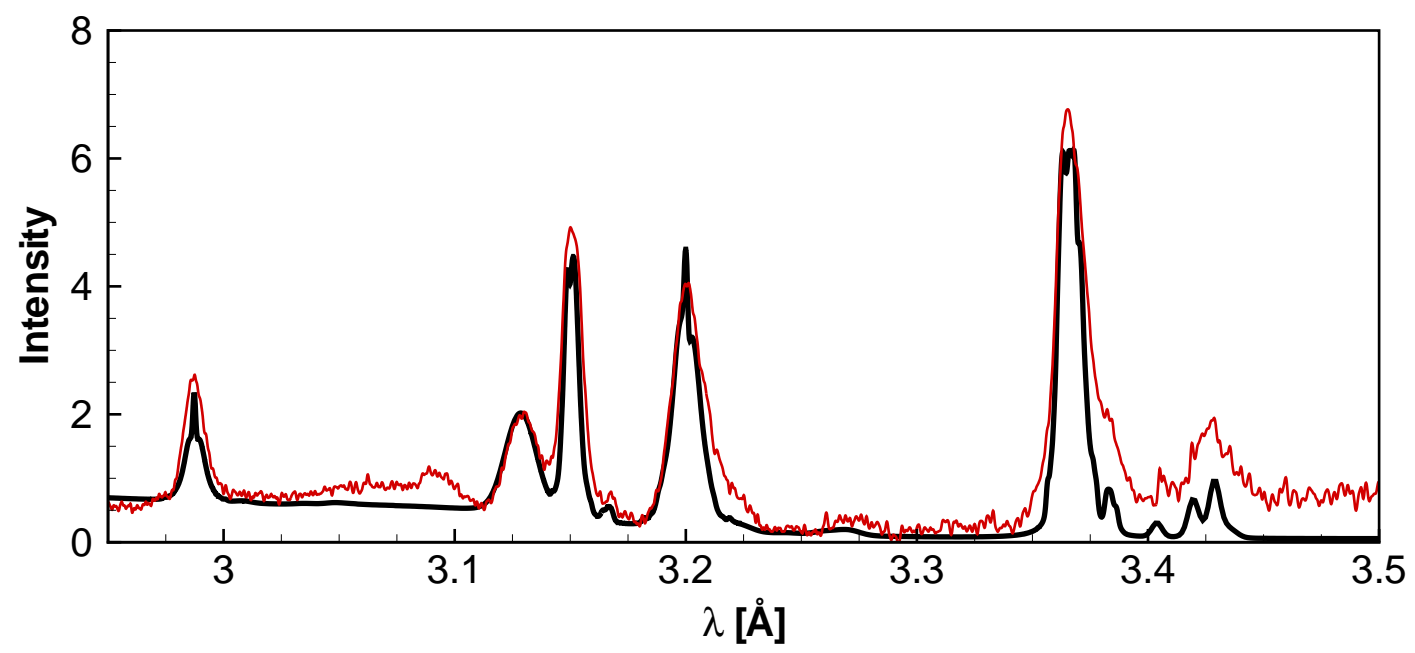

Fig. 6. 